Mon. Not. R. Astron. Soc. (to be submitted), 田囵(2006) Printed 17 June $2018 \quad$ (MN LATEX style file v2.2)

\title{
Persistent Patterns in Accretion Disks
}

\author{
Mustafa A. Amin and Andrei V. Frolov ${ }^{\star}$ \\ KIPAC, Stanford University, Stanford, CA, 94305-4060
}

24 March 2006

\begin{abstract}
We present a set of new characteristic frequencies associated with accretion disks around compact objects. These frequencies arise from persistent rotating patterns in the disk that are finite in radial extent and driven purely by the gravity of the central body. Their existence depends on general relativistic corrections to orbital motion and, if observed, could be used to probe the strong gravity region around a black hole. We also discuss a possible connection to the puzzle of quasi-periodic oscillations.
\end{abstract}

Key words: black hole physics - accretion, accretion discs

\section{INTRODUCTION}

Timing observations of accreting X-ray binary systems have revealed luminosity modulation at a number of characteristic frequencies. Phenomenology of these quasi-periodic oscillations (QPOs) is quite rich. For a detailed review, see McClintock \& Remillard (2003) and references therein. Some of the features are rather puzzling, such as stability of high frequency QPOs in black hole binaries and that in some systems they appear in pairs at 3:2 frequency ratio.

QPOs in black hole systems are thought to arise from physical processes in accretion disks. Depending on where the oscillations reside, one can roughly divide models for QPOs in accretion disks into two classes: local and global. Local models tie down the oscillation frequency to a particular place in the disk (like an edge or a hot spot). In this case, the question of what determines that place has to be answered. One line of argument is that the location of the hot spot is determined by a resonance (Abramowicz \& Kluźniak 2001; Török et al. 2005; Kluźniak 2005; Abramowicz 2005). This model has an attractive feature that the observed 3:2 frequency ratio can be explained by non-linear mode locking. A hot spot can give rise to luminosity variation, for example, due to Doppler beaming (Schnittman \& Bertschinger 2004; Schnittman 2005). However, a potential difficulty is to have a hot spot which is sufficiently bright. Achieving sufficient luminosity variation seems less problematic in global models, in which modes occupy a larger region of the disk. Linear perturbation analysis of the accretion disk in diskoseismology approach (Wagoner et al. 2001; Perez et al. 1997; Silbergleit et al. 2001; Ortega-Rodríguez et al. 2002) naturally solves the issue of spatial and frequency localization of modes. The 3:2 frequency ratio would be accidental for two fundamental diskoseismic modes, but it could arise from higher azimuthal $g$-modes which are nearly harmonic.

* E-mail: mamin@stanford.edu, afrolov@stanford.edu
In this paper, we describe a set of new characteristic frequencies which might be present in accretion disks around compact objects. To the best of our knowledge, they have remained unnoticed in the literature. These frequencies arise from rotating patterns in the disk which are quasistationary, finite in radial extent, and driven purely by gravity of the central body. We neglect self-gravity and the hydrodynamics of the accreting matter. The main idea is similar to the notion of density waves that give rise to the spiral structure in galaxies (Lindblad 1963; Lin \& Shu 1964, 1966), although these patterns depend on general relativity rather than a distributed matter source for their existence.

While it is tempting to identify the frequencies of these patterns with the source of QPOs, we cannot claim to have a complete model. The issues of how they are excited, how they translate to X-ray luminosity variation, and effects of pressure and viscosity need to be investigated in more detail. We will return to these points with some plausibility arguments in Section 4

\section{ACCRETION DISK KINEMATICS}

A test particle in a circular equatorial orbit around a Kerr black hole has an orbital frequency (Bardeen et al. 1972)

$\Omega=\frac{1}{r^{3 / 2}+a}$

with respect to Boyer-Lindquist time $t$, where $r$ is the orbit radius and $a$ is the dimensionless black hole spin parameter $\left(a=c J / G M^{2}\right)$. We work in dimensionless units scaled by the black hole mass $M$ (i.e., distances measured in units of $G M / c^{2}$, times measured in units of $G M / c^{3}$, etc.), and will further set $G=c=1$. Here and later we will assume that particles co-rotate with the black hole.

If perturbed from the circular orbit, the particle will undergo radial and perpendicular oscillations with epicyclic frequencies $\kappa$ and $\Omega_{\perp}$ respectively (Okazaki et al. 1987) 


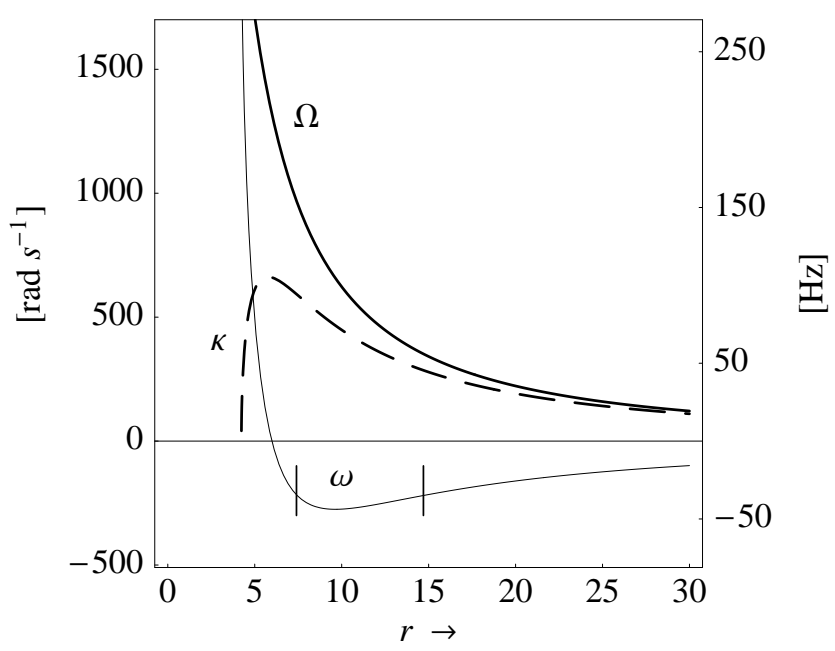

Figure 1. Orbital frequency $\Omega$, radial epicyclic frequency $\kappa$, and precession frequency $\omega=\Omega-2 \kappa$ of a $2: 1$ orbit in an accretion disk around a Kerr black hole with $M=10 M_{\odot}$ and $a=1 / 2$. Precession frequency exhibits a shallow negative minimum at $r_{*} \approx 9.64$. The radial extent of the rotating pattern with $20 \%$ deviation in frequency is marked by vertical bars.

$\kappa^{2}=\Omega^{2}\left(1-\frac{6}{r}+\frac{8 a}{r^{3 / 2}}-3 \frac{a^{2}}{r^{2}}\right)$

and

$\Omega_{\perp}^{2}=\Omega^{2}\left(1-\frac{4 a}{r^{3 / 2}}+3 \frac{a^{2}}{r^{2}}\right)$.

The factors multiplying $\Omega$ on the right-hand sides of these expressions are general relativistic corrections. They are absent in Keplerian mechanics, where both epicyclic and orbital frequencies are all the same $\left(\kappa=\Omega_{\perp}=\Omega\right)$. The radial dependences of orbital frequency $\Omega$ and radial epicyclic frequency $\kappa$ for a typical rotating black hole are illustrated in Figure 1 Circular orbits close to a black hole are unstable; the innermost stable circular orbit (ISCO) is located where $\kappa^{2}$ vanishes.

If orbital and epicyclic frequencies are the same, as they are for a Keplerian potential, the orbits are closed. However, if the potential deviates from $1 / r$ (either because of general relativity corrections, as in our case, or due to a distributed matter source, as happens in galaxies), the two frequencies will in general be different, and the orbits will precess. The condition for an orbit to close in a frame rotating with frequency $\omega$ is for the orbital and epicyclic frequencies to be commensurate, $m(\Omega-\omega)=n \kappa$, which gives the precession frequency

$\omega=\Omega-\frac{n}{m} \kappa$.

The integers $n$ and $m$ determine the shape of the precessing orbit, and from here on, we will use the abbreviation $n: m$ to refer to their values. Figure 2 shows the shape of $1: 2$ and $2: 1$ orbits, which are representative of the deformed and the self-intersecting orbit classes.

In general, precession frequency $\omega$ depends on $r$, and any pattern initially present will shear away as the disk rotates. However, if $\omega$ is approximately constant over some portion of the disk, collective orbit precession can lead to a nearly rigid pattern rotation. One example of this is the spiral structure in galaxies caused by the 1:2 mode (Lindblad 1963; Lin \& Shu 1964, 1966). The 1:1 mode in Keplerian disks gives rise to a static one-armed spiral pattern (Kato 1983), which is seen in numerical simulations as well (Havasaki \& Okazaki 2005). An attempt has been made to trap the 1:1 mode in the region of the strong gravity (Kato 1990), but trapping depends strongly on the pressure distribution within the disk (Kato \& Honma 1991).

\section{PERSISTENT PATTERNS IN THE DISK}

Our key observation is that for $n>m$, the precession frequency $\omega(r)$ develops a very shallow minimum at a radius $r=r_{*}$, as illustrated in Figure 1 1 for the 2:1 orbit. Collective excitation of particles on orbits precessing at the same rate would lead to a pattern occupying a sizable portion of the disk around $r_{*}$ and rotating with little shear at a frequency $\omega_{p}=\omega\left(r_{*}\right)$. Somewhat unusual are the facts that the pattern is counter-rotating and that the orbit closes in several rotations rather than a single one.

In a real accretion disk, the collective particle motion would have to be excited by some dynamical mechanism. It might be complicated and could require numerical simulations of the disk to fully understand the driving process. In the present paper, we will be content with studying the kinematics only. We will set off a collective mode by selecting appropriate initial conditions (as described below) and follow the pattern evolution by tracing the motion of individual particles making up the disk. The purpose is to find out if there is a long-lived pattern that survives the differential rotation.

If perturbed from a circular orbit at $r=r_{0}$ by a small displacement $\varepsilon$ in the radial direction, the trajectory of a test particle (to first order in $\varepsilon$ ) is

$r(t)=r_{0}+\varepsilon \sin (\kappa t+\chi)$,

$\phi(t)=\varphi+\Omega t+\frac{2 \Omega}{\kappa r} \Upsilon \varepsilon \cos (\kappa t+\chi)$,

where $\varphi$ and $\chi$ are initial orbital and epicyclic phases, and

$\Upsilon=\frac{1-\frac{3}{r}+\frac{2 a}{r^{3 / 2}}}{1-\frac{2}{r}+\frac{a^{2}}{r^{2}}}\left(r^{3 / 2} \Omega\right)$

is a relativistic correction factor (which, however, changes little in the region of the disk we are interested in). We populate the disk by spreading $N$ particles uniformly on a $n: m$ orbit, with initial phases of a $k^{\text {th }}$ particle

$\varphi_{k}=\frac{2 \pi n}{N} k, \quad \chi_{k}=\frac{2 \pi m}{N} k$,

while stacking the orbits in a radial direction at an angle $\alpha$ by giving the orbit located at $r_{j}$ a phase offset

$\varphi_{j k}=\varphi_{k}+\alpha r_{j}$

This particle distribution leads to a spiral structure in the disk. Figure 3 shows the surface density contrast (smoothed with a Gaussian kernel) for patterns obtained by distributing particles on stacked 1:2 (left) and 2:1 (right) orbits. The three rows of Figure 3 show a time-lapse sequence of pattern evolution. The top row shows the initial conditions, and the second and the third rows show patterns at $t=T_{2: 1}$ and 

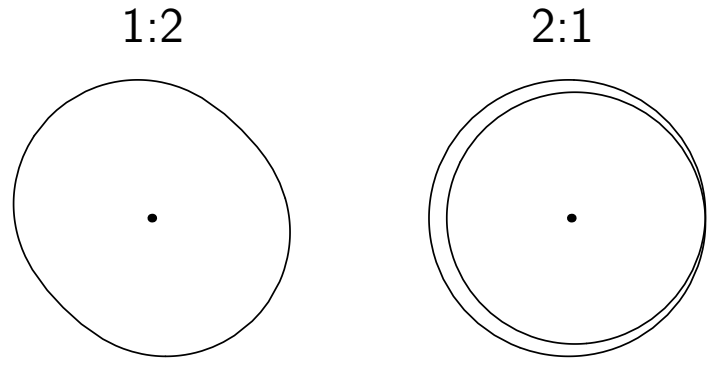

Figure 2. Sample closed orbits. 1:2 orbit (left) corresponds to 2 epicycles per 1 rotation around a central body, while 2:1 orbit (right) corresponds to 1 epicycle per 2 rotations.

$1: 2$
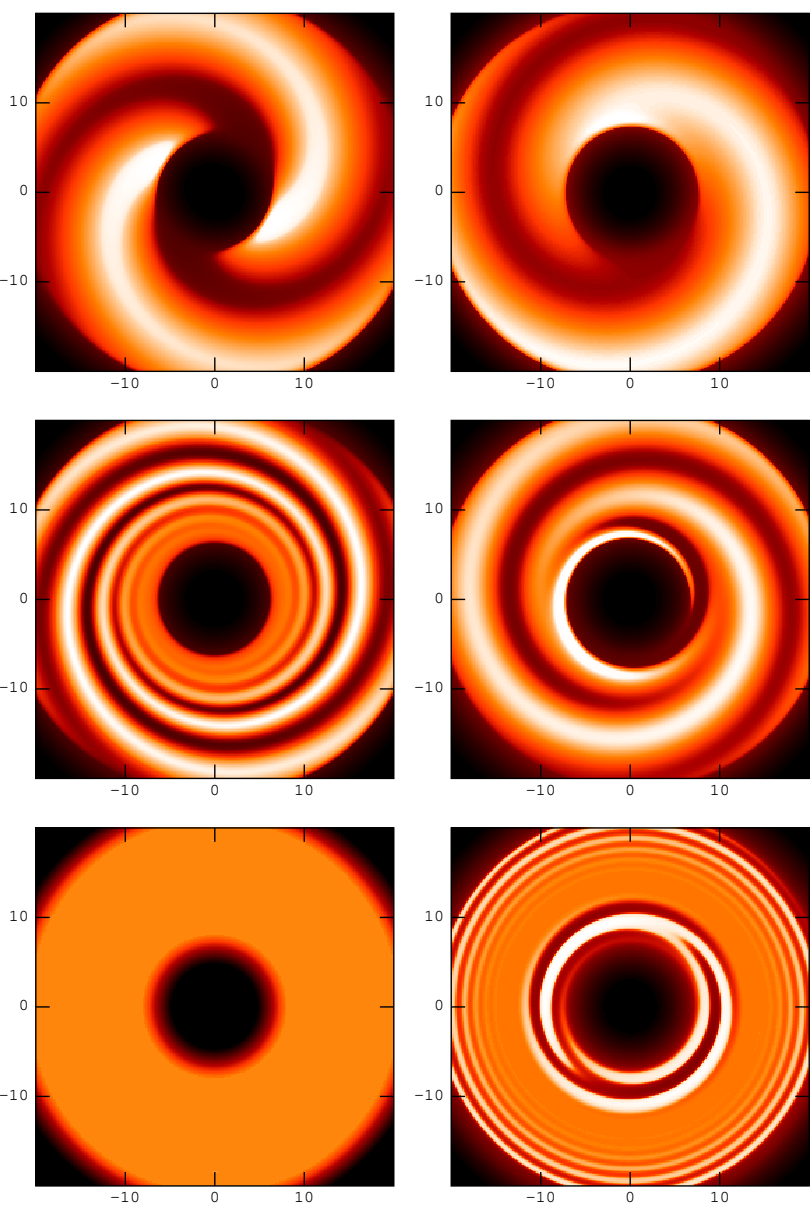

Figure 3. Evolution of density patterns obtained by distributing particles on stacked 1:2 (left) and 2:1 (right) orbits. Top row shows initial configuration, middle row - after one period of rotation of 2:1 pattern elapsed, bottom row - after twenty periods. Density contrast has been enhanced by histogram equalization.

$t=20 T_{2: 1}$ correspondingly. $T_{2: 1}=2 \pi / \omega_{2: 1}$ denotes a period of rotation of the $2: 1$ pattern.

The frequency of the 1:2 orbit precession depends monotonically on the radius, so one expects differential rotation to destroy the pattern. Indeed, at $t=T_{2: 1}$, the spiral is seen to wind up, and by $t=20 T_{2: 1}$, it is wound up so tightly that the smoothing removes all traces of structure. The evolu- tion of the 2:1 pattern is markedly different. Signs of shear are clearly seen after a single rotation. However, even after twenty rotations, there is still a pattern present around $r_{*} \approx 9.6$ (which is exactly where the minimum of $\omega_{2: 1}$ occurs). As this time span corresponds to almost 50 orbital rotations at $r_{*}$, the pattern is remarkably persistent.

\section{DISCUSSION}

In the last section, we have shown that an accretion disk around a compact object can support persistent rotating patterns due to the collective excitations of particles in the disk. Their existence depends on general relativity effects and is sensitive to the parameters of the central body but not to the accretion rate. All the frequencies in the problem scale inversely proportionally to the central body mass. In addition, persistent pattern frequencies depend on the spin parameter. Figure 4 shows the rotation frequencies of the three lowest-order persistent patterns (2:1, 3:1, and 3:2) for a $10 M_{\odot}$-mass black hole as the spin is varied. This dependence in principle could be used to measure the mass and spin of the central object, provided that the frequencies of two distinct modes are observed and identified correctly. One should note, though, that for multi-armed patterns (for example the 3:2 pattern which has two arms) modulation frequency could be a multiple of the rotation frequency.

Several different persistent patterns could coexist in the accretion disk; however, it is likely that the lowest-order ones are strongly selected based on geometrical considerations. Surface density modulation of the $2: 1$ pattern is secondorder in particle displacement $\varepsilon$, while the $3: 1$ and $3: 2$ patterns are third-order. The cancellation of lower-order terms is directly caused by the multiple-fold geometry of the orbits with $n>1$. Being higher-order could explain why these persistent patterns are not apparent in the linear perturbation analysis of Wagoner et al. (2001). Despite second-order scaling, the 2:1 pattern in Figure 3 (lower right) shows $1 \%$ amplitude of the surface density modulation for moderate displacement values $\left(\varepsilon / r_{*} \approx 0.022\right)$. The radial extent of the 2:1 pattern $(\Delta r \sim 4)$ also appears to be wider than that of a fundamental $g$-mode (the width of which is proportional to $c_{s}^{1 / 2}$ and is estimated as $\Delta r \sim 1$ by Wagoner et al. (2001)).

In this paper, we focused on kinematics and neglected particle interactions and the hydrodynamics of the disk. The extent to which this approximation is justified should be further investigated. Of critical importance for the model is understanding the excitation mechanism. It is possible for the spiral waves to be driven from the outer edge of the disk (Spruit 1987; Spruit et al. 1987); however, whether that is sufficient to cause persistent patterns to appear remains to be seen. Both questions could be answered by turning to numerical simulations of the accretion disk hydrodynamics. However, that is a much more complicated problem, and we feel that it is beyond the scope of this paper, the intent of which is merely to point out the existence of new characteristic frequencies in the disk.

It is plausible that the characteristic frequency of the collective motion will manifest itself in X-ray luminosity variation, but the exact mechanism responsible for the modulation is not clear to us. Density pattern in the accretion disk need not be a direct cause. Particles weaving in and out 


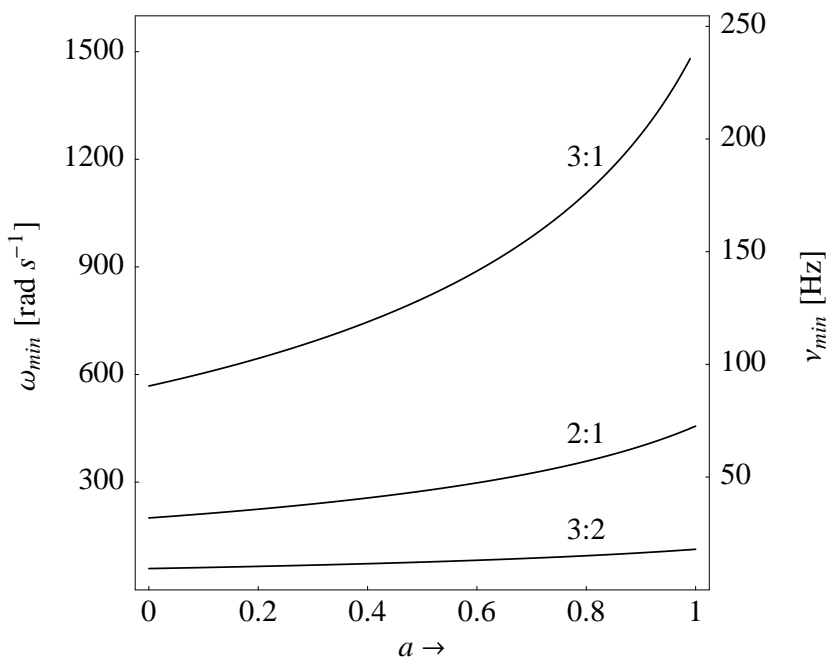

Figure 4. Persistent pattern frequencies of three lowest-order radial modes $(2: 1,3: 1$, and $3: 2)$ for a $10 M_{\odot}$-mass black hole as a function of black hole spin parameter $a$.

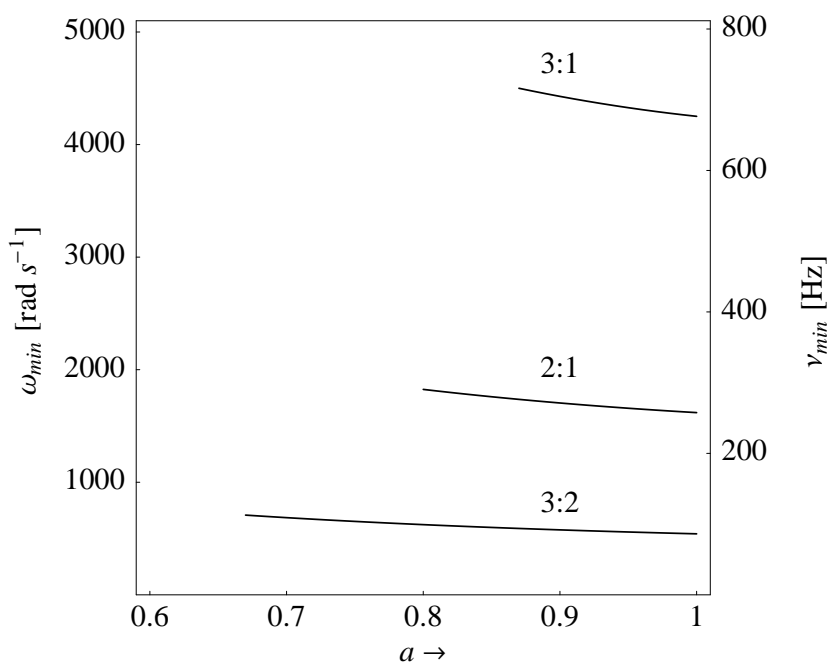

Figure 5. Persistent pattern frequencies of three lowest-order transverse modes $(2: 1,3: 1$, and $3: 2)$ for a $10 M_{\odot}$-mass black hole as a function of black hole spin parameter $a$.

on self-intersecting orbits could lead to efficient gas heating, possibly due to shock formation, and create a temperature pattern in the accretion disk (in a sense, an extensive "hot spot"). The picture of temperature modulation of the disk causing X-ray luminosity variations is not entirely satisfactory as quasi-periodic oscillations are seen primarily in the hard non-thermal component of the emission (McClintock \& Remillard 2003). That could indicate that the quasi-periodic emission is coming from a coronal region rather than from a disk (Titarchuk \& Fiorito 2004). It is possible that the transfer mechanism might involve a magnetic field threading the disk (Blandford, unpublished).

So far we have been talking about patterns arising from radial oscillations. It is worth mentioning that a similar thing could happen for transverse oscillations as well. The precession frequency $\omega_{\perp}=\Omega-\frac{n}{m} \Omega_{\perp}$ also has a minimum if $n>m$. However, the minimum lies inside an innermost stable circu- lar orbit unless the black hole is spinning rapidly ( $a>0.8$ for $2: 1$ orbit). The frequencies of the three lowest-order transverse modes are shown in Figure 5 Transverse particle excitations would lead to a corrugated accretion disk rather than a surface density pattern.

To summarize, we have found a set of new characteristic frequencies associated with accretion disks around compact objects. Although many questions remain, it might be interesting to pursue this idea further and see if it could lead to a model of quasi-periodic oscillations in X-ray binaries. In particular, the numerical values of our characteristic frequencies and their independence of the accretion rate suggest an application to high-frequency QPOs in black hole binaries.

\section{ACKNOWLEDGMENTS}

We would like to thank Andrei Beloborodov, Roger Blandford, Steven Fuerst, Włodek Kluźniak, and Robert Wagoner for helpful discussions. AF is supported in part by the Stanford Institute for Theoretical Physics.

\section{REFERENCES}

Abramowicz, M. A. 2005, Astron. Nachr., 326, 782

Abramowicz, M. A., \& Kluźniak, W. 2001, A\&A, 374, L19

Bardeen, J. M., Press, W. H., \& Teukolsky, S. A. 1972, ApJ, 178, 347

Hayasaki, K., \& Okazaki, A. T. 2005, Mon. Not. R. Astron. Soc., 360, L15

Kato, S. 1983, Publ. Astron. Soc. Japan, 35, 249

Kato, S. 1990, Publ. Astron. Soc. Japan, 42, 99

Kato, S., \& Honma, F. 1991, Publ. Astron. Soc. Japan, 43, 95

Kluźniak, W. 2005, Astron. Nachr., 326, 820

Lin, C. C., \& Shu, F. H. 1964, ApJ, 140, 646

Lin, C. C., \& Shu, F. H. 1966, Proc. Nat. Acad. Sci., 55, 229

Lindblad, B. 1963, Stockholms Observ. Ann., 22, 3

McClintock, J. E., \& Remillard, R. A. 2003, arXiv:astro-ph/0306213

Okazaki, A. T., Kato, S., \& Fukue, J. 1987, Publ. Astron. Soc. Japan, 39, 457

Ortega-Rodríguez, M., Silbergleit, A. S., \& Wagoner, R. V. 2002, ApJ, 567, 1043

Perez, C. A., Silbergleit, A. S., Wagoner, R. V., \& Lehr, D. E. 1997, ApJ, 476, 589

Schnittman, J. D. 2005, ApJ, 621, 940

Schnittman, J. D., \& Bertschinger, E. 2004, ApJ, 606, 1098

Silbergleit, A. S., Wagoner, R. V., \& Ortega-Rodríguez, M. 2001, ApJ, 548, 335

Spruit, H. C. 1987, A\&A, 184, 173

Spruit, H. C., Matsuda, T., Inoue, M., \& Sawada, K. 1987, Mon. Not. R. Astron. Soc., 229, 517

Titarchuk, L., \& Fiorito, R. 2004, ApJ, 612, 988

Török, G., Abramowicz, M. A., Kluźniak, W., \& Stuchlík, Z. 2005, A\&A, 436, 1

Wagoner, R. V., Silbergleit, A. S., \& Ortega-Rodríguez, M. 2001, ApJ Lett., 559, L25 